\title{
Curvas de aprendizado: estado da arte e perspectivas de pesquisa
}

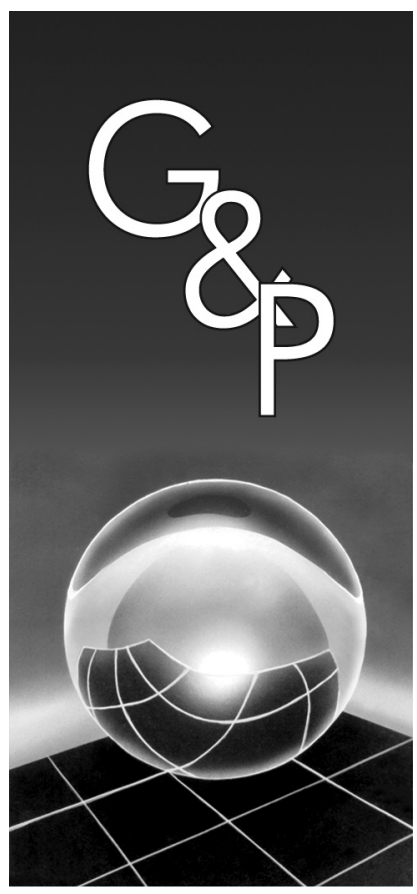

Michel José Anzanello

Flávio Sanson Fogliatto

\section{Resumo}

Curvas de aprendizado têm se mostrado ferramentas úteis no monitoramento do desempenho de um trabalhador submetido a uma nova tarefa, avaliando seu progresso na medida que repetições são efetuadas. Essas curvas foram introduzidas por Wright em 1936, e desde então, têm sido utilizadas para avaliação do tempo demandado para a conclusão de corridas de produção, estimação da redução de custos de produção e alocação de trabalhadores para tarefas com base em suas características de atuação. Este artigo apresenta o estado da arte da literatura em torno do tema, abordando os modelos existentes, aplicações em contextos práticos e limitações da ferramenta. Por fim, direcionamentos para pesquisas futuras serão propostos.

Palavras-chave: Curvas de aprendizado. Desempenho do trabalhador. Programação da produção.

\section{Introdução}

O processo de aquisição de conhecimento e destreza tem sido estudado em várias perspectivas e com diferentes objetivos. Em busca da melhoria contínua dos meios produtivos, pesquisadores da área de Engenharia têm sugerido diversas sistemáticas para explicar o aprimoramento resultante da repetição de tarefas, bem como os fatores que influenciam tal progresso (ANDERSON, 1982; NEMBHARD; OSOTHSILP, 2002; NEMBHARD; UZUMERI, 2000a; PANANISWAML; BISHOP, 1991; ADLER; CLARK, 1991; VITS; GELDERS, 2002). Dentre os fatores contemplados na literatura, merecem destaque: i) política de treinamento adotada pela empresa (TERWIESCH; BOHN, 2001; VITS; GELDERS, 2002; SEREL et al., 2003), ii) motivação do trabalhador em realizar as tarefas demandadas (KANFER, 1990; EYRING; JOHNSON; FRANCIS, 1993; NATTER et al., 2001; AGRELL; BAGETOFT; TIND, 2002), iii) existência de conhecimento prévio (experiência) na execução da tarefa (NEMBHARD; UZUMERI, 2000a, 2000b; NEMBHARD; OSOTHSILP, 2002); e iv) complexidade da tarefa (PANANISWAML; BISHOP, 1991; NEMBHARD; OSOTHSILP, 2002). Outros estudos abordam fatores como destreza e retenção de conheci- mento, quando o trabalhador interrompe temporariamente a execução de uma tarefa (DAR-EL; RUBINOVITZ, 1991; WICKENS; GORDON; LIU, 1998; NEMBHARD; UZUMERI, 2000b). Na quase totalidade dos estudos, o efeito dos diferentes fatores sobre o processo de aprendizagem é descrito por modelos matemáticos, propostos para essa finalidade.

A curva de aprendizado apresenta-se como uma ferramenta capaz de monitorar o desempenho de trabalhadores submetidos a tarefas repetitivas. Através das curvas é possível analisar e programar tarefas produtivas, reduzindo perdas decorrentes da inabilidade do trabalhador, as quais são verificadas principalmente nos primeiros ciclos de produção (ARGOTE, 1999; DAR-EL, 2000). A ferramenta também permite a adequada alocação de tarefas aos membros de uma população de trabalhadores, obedecendo suas características de atuação (TEPLITZ, 1991; UZUMERI; NEMBHARD, 1998; NEMBHARD; UZUMERI, 2000a; ANZANELLO; FOGLIATTO, 2006), além de permitir o monitoramento dos custos ligados ao processo de aprendizado (WRIGHT, 1936; TEPLITZ, 1991; STURM, 1999). 
Dada a vasta utilização das curvas de aprendizado em aplicações práticas e o grande número de publicações em torno do tema, este artigo traz uma revisão do estado da arte da literatura sobre o tema, apresentando os modelos e os principais cenários de utilização. Duas contribuições se destacam no presente artigo: i) a descrição da estrutura matemática de curvas de aprendizado univariadas e multivariadas, abordando suas aplicações, modificações e limitações; e ii) a apresentação de linhas de pesquisa e sugestões para trabalhos futuros sobre o tema.

Este artigo está estruturado da seguinte forma: a seção 2 apresenta a fundamentação teórica em torno dos modelos de curvas de aprendizado, com subseções devotadas às principais famílias de modelos, a seção 3 traz sugestões para pesquisas futuras sobre curvas de aprendizado, enfocando aspectos práticos e teóricos sobre o tema e a conclusão encerra o artigo na seção 4 .

\section{Definição e modelos de curvas de aprendizado: estado da arte}

Curvas de aprendizado são representações matemáticas do desempenho de um trabalhador quando submetido a uma tarefa manual repetitiva (WRIGHT, 1936; TEPLITZ, 1991; BADIRU, 1992; ARGOTE, 1999). À medida que repetições são efetuadas, o trabalhador demanda menos tempo para a execução da tarefa, seja pela familiaridade adquirida com os meios de produção, seja pela adaptação às ferramentas utilizadas ou pela descoberta de "atalhos" para realização da tarefa (WRIGHT, 1936; TEPLITZ, 1991; DAR-EL, 2000).

O desenvolvimento seminal das curvas de aprendizado deve-se a Wright (1936), sendo resultante da observação da redução no custo da montagem de aviões durante a Primeira Guerra Mundial. Essa redução obedecia a uma taxa constante, com a duplicação da quantidade de aviões produzidos, levando Wright a formular uma regra prática, denominada "curva de 80\%", para a indústria aeronáutica da época. Segundo essa regra, a montagem de determinada quantidade de aeronaves sofria redução de $20 \%$ no custo acumulado médio a cada duplicação da quantidade produzida (TEPLITZ, 1991; COOK, 1991; BADIRU, 1992; ARGOTE, 1999; ASKIN; GOLDBERG, 2001).

A medição do desempenho do trabalhador na execução de uma tarefa pode ser feita através da observação das seguintes variáveis de resposta: i) tempo de trabalho demandado por unidade fabricada; ii) número de unidades fabricadas em intervalo fixo de tempo; iii) redução dos custos do produto por unidade; e iv) porcentagem de unidades não-conformes pelo número de repetições de uma mesma operação (TEPLITZ, 1991; FRANCESCHINI; GALETTO, 2002).

Os parâmetros constituintes das curvas de aprendizado são obtidos através de regressão não-linear por intermédio de software de análise estatística, buscandose a minimização da soma quadrada dos erros. Casos em que a modelagem não apresenta convergência imediata, comuns na regressão não-linear, podem ser solucionados através da modificação nos valores iniciais dos parâmetros. A aderência do modelo pode ser avaliada por intermédio do coeficiente de Determinação $\left(R^{2}\right)$, pela Soma Quadrada dos Erros ou pelo desvio do modelo gerado frente a uma amostra de validação.

A aplicação de curvas de aprendizado no monitoramento e avaliação do desempenho de trabalhadores ocasionou o desenvolvimento de modelos distintos de curvas univariadas e multivariadas. Esses modelos são constituídos por funções matemáticas de complexidades diversas, possibilitando a descrição do processo de aprendizado em diversos setores. Dentre as curvas de aprendizagem univariadas mais difundidas, merecem destaque os modelos potenciais, exponenciais e hiperbólicos, apresentados na sequência.

\subsection{Modelos potenciais}

Dado seu pioneirismo, o modelo de Wright é formalmente conhecido como "modelo potencial", fazendo com que outros modelos baseados em curvas matemáticas desta natureza possuam denominações específicas. A curva é representada por:

$$
y=C_{1} x^{b}
$$

onde, $y$ indica o tempo (custo) médio por unidade demandado para a produção de $x$ unidades e $C_{1}$ é o tempo (custo) da primeira unidade produzida. O parâmetro $b$ (declividade da curva de aprendizado) é gerado por intermédio de manipulação matemática da taxa de aprendizado do trabalhador (medida percentualmente), sendo seu valor compreendido no intervalo -1 a 0 . Valores de $b$ próximos da unidade negativa indicam um elevado percentual de aprendizado e assimilação rápida dos preceitos da tarefa (TEPLITZ, 1991; BADIRU, 1992; ARGOTE, 1999; DAR-EL, 2000).

O modelo de Wright pode ser manipulado de forma a permitir o cálculo do tempo (custo) total demandado para fabricação de $x$ unidades através da Equação 2:

$$
y_{1 \rightarrow x}=C_{1} x^{b+1}
$$

e do tempo (custo) necessário para fabricação de uma unidade específica $x$, por intermédio da Equação 3:

$$
y_{x}=C_{1}\left[x^{b+1}-(\mathrm{x}-1)^{b+1}\right]
$$

Tais relações encontram-se compiladas em tabelas baseadas na taxa percentual de aprendizado, permitindo a obtenção dos resultados de maneira direta (WRIGHT, 1936; TEPLITZ, 1991).

Por conta de sua flexibilidade, o modelo potencial tem sido utilizado na elaboração de estratégias de produção 
(KORTGE, 1993), estimativas de tempo para conclusão de determinadas tarefas (TEPLITZ, 1991), verificação do efeito de paradas no processo produtivo (JABER; BONNEY, 1996; ARGOTE, 1999) e levantamento das consequiências das modificações nos parâmetros de um processo durante a sua execução (TOWILL, 1985). Dentre os setores que fazem uso da curva de aprendizado potencial, merecem destaque a indústria de semicondutores (COOK, 1991; GRUBER, 1994, 1996, 1998), calçados (JACINTO, 2001), construção civil (LEITE, 2002), eletrônicos e aeroespacial (GARVIN, 2000), automotiva (DAR-EL, 2000) e de montagem de caminhões (ARGOTE, 1999). Exemplos da aplicação de curvas potenciais no monitoramento de custos podem ser obtidos em Teplitz (1991), Rea e Kerzner (1997) e Teng e Thompson (1996).

Para Blancett (2002) e Globerson e Gold (1997), a curva potencial é incontestavelmente o modelo de curva de aprendizado mais utilizado para previsão de produtividade. Segundo Globerson e Levin (1987) e Vits e Gelders (2002), a curva pode ser utilizada para descrever com boa precisão a maior parte das tarefas que se baseiam em procedimentos repetitivos, além de apresentar a vantagem de ser matematicamente simples. Blancett (2002) utilizou-a em diversos setores de uma empresa de manufatura de material de construção, abrangendo desde etapas do desenvolvimento de produto até a produção em larga escala, enquanto que Terwiesch e Bohn (2001) avaliaram o aprimoramento de trabalhadores no intervalo entre o início da produção de um novo produto e a plena utilização da capacidade produtiva. Indicadores produtivos de layouts celular e de processo foram comparados por Kannan e Palocsay (1999) através de modificações na curva de aprendizado potencial.

Curvas de aprendizado potenciais também foram largamente utilizadas no setor de serviços. Chambers e Johnston (2000) aplicaram a ferramenta em duas fornecedoras de serviços: uma grande empresa de transporte aéreo e uma pequena instituição bancária, enquanto que Saraswat e Gorgone (1990) monitoraram o desempenho de equipes de trabalhadores responsáveis pela instalação de softwares em empresas e domicílios. Sturm (1999) verificou reduções nos custos de serviços clínicos na ordem de $15 \%$ com a duplicação da experiência, essa, medida em número de formulários preenchidos dos funcionários responsáveis pelo procedimento.

A programação de produção também pode ser auxiliada por curvas de aprendizado potenciais. Kopcso e Nemitz (1983) verificaram a existência de custos decorrentes da não consideração do aprendizado dos trabalhadores na determinação do tamanho ideal de lote de produção. Por conta disso, Muth e Spremann (1983) e Jaber e Bonney (1999) desenvolveram equações para obtenção do tamanho ótimo de lotes de produção, em situações nas quais se verifica aprendizado. Salameh, Abdul-Malak e Jaber (1993) propuseram um método simplificado para analisar a influência do aprendizado sobre o tamanho do lote ótimo de produção e custo de estoque. O método incorpora a curva potencial para obtenção do custo de produção. Em estudos similares, Jaber e Bonney (1999, 2001, 2003) analisaram a magnitude das alterações no tamanho do lote ideal e nos custos de estoque, decorrentes do aprendizado no processo de produção. Desenvolvendo modelos próprios de curva de aprendizado, Rachamadugu e Tan (1997) e Pratsini (2000) também avaliaram os impactos do aumento de destreza sobre o tamanho ideal dos lotes de produção.

Buscando vincular a curva de aprendizado às ferramentas para controle de qualidade, Koulamas (1992) desenvolveu uma metodologia capaz de avaliar o efeito do redesign de produtos sobre variáveis de qualidade e custo do processo, enquanto que Teng e Thompson (1996) analisaram o efeito do aprendizado sobre qualidade e custos de um novo produto na indústria automotiva. Por sua vez, Franceschini e Galetto (2002) propuseram um método para obtenção de uma estimativa preliminar da redução do percentual de produtos não-conformes, em uma planta de produção de suco.

Curvas de aprendizado potenciais também têm sido utilizadas em conjunto com outras ferramentas destinadas ao controle de produção. Yelle (1980), Kortge (1993) e Kortge et al. (1994) sugeriram a utilização das curvas em conjunto com o Modelo do Ciclo de Vida do Produto, descrito em Cox (1967) e em Rink e Swan, (1979), dentre outros, objetivando um planejamento mais preciso da produção. Em estudo posterior, Yelle (1983) utilizou tais ferramentas na linha de montagem do carro Ford-T, analisando a redução do valor dos custos de peças e montagem com o aumento de unidades fabricadas. Pramongkit, Shawyun e Sirinaovakul (2000) utilizaram a curva potencial vinculada à função de produção de Cobb-Douglas, analisando a importância de fatores como: capital investido e nível de mão-de-obra especializada empregada no aprendizado dos trabalhadores de indústrias tailandesas. Seguindo o mesmo propósito de pesquisa, Pramongkit, Shawyun e Sirinaovakul (2002) aplicaram o modelo potencial em conjunto com um indicador de crescimento tecnológico e produtivo (Total Factor Productivity - TFP), avaliando a influência do grau de desenvolvimento tecnológico de grandes empresas tailandesas sobre o potencial de aprendizado dos trabalhadores. Por fim, Karaoz e Albeni (2005) propuseram um modelo baseado em curvas de aprendizado e indicadores tecnológicos capaz de estimar o aprendizado dos trabalhadores durante longos períodos de operação em empresas turcas.

Por se tratar do modelo mais simples e difundido entre as curvas de aprendizado, a curva potencial tem sido 
investigada no que se refere às suas limitações e adaptações para cenários específicos (ZANGWILL; KANTOR, 1998, 2000; WATERWORTH, 2000). As adaptações buscam a eliminação de inconsistências decorrentes da simplicidade matemática da equação potencial.

Hurley (1996) e Eden, Willians e Ackermann (1998) alegam que o modelo proposto por Wright, quando utilizado em ciclos longos de produção, resulta em predições de tempo de execução da tarefa próximas ao zero, o que não se verifica na prática. Para tanto, sugerem a inclusão de um parâmetro no modelo para contornar tal inconsistência. Outra limitação, segundo Globerson, Levin e Shtub (1989), provém do fato do modelo de Wright não fazer referência à experiência anterior do trabalhador em uma operação, fator que determina um rendimento superior deste trabalhador em relação a outro que nunca executou a mesma operação.

De acordo com Towill $(1985,1990)$ e Waterworth (2000), a larga utilização de curvas de aprendizado como ferramenta de previsão deu margem a definições confusas e incorretas de seus parâmetros, citando como exemplos: os freqüentes equívocos entre tempo médio e tempo específico para execução da unidade $x$. Por conta disso, Smunt (1999) sugeriu uma interpretação alternativa para o conceito de repetição, o que eliminaria tais equívocos. As repetições passariam a ser computadas como metade do número de unidades componentes da corrida de produção (repetição $i / 2$, no caso de corridas com $i$ unidades). Essa forma de representação é justificada pela Teoria de Aprendizado Contínuo proposta por Smunt (1999), segundo a qual o aprendizado ocorre durante a execução da repetição e não quando a repetição foi concluída.

A variabilidade dos dados de desempenho coletados dos processos pode gerar curvas com ajustes deficientes e baixo poder preditivo (YELLE, 1979; GLOBERSON, 1984; VIGIL; SARPER, 1994) afirmam que a estimativa inconsistente da declividade de aprendizado (parâmetro b) constitui-se a principal causadora de discrepâncias, levando-os a sugerir adaptações (no modelo potencial) capazes de gerar intervalos de confiança para os tempos previstos para execução da tarefa. Globerson e Gold (1997) desenvolveram expressões para obtenção da variância, coeficiente de variação e função-densidade de probabilidade do desempenho predito pela curva de aprendizado. Complementarmente, Smunt (1999) sugeriu modificações no modelo potencial com o propósito de adaptar a curva de aprendizado às situações nas quais se verificam alterações no valor da declividade de aprendizado, no decorrer do processo produtivo (característica de operações em bateladas). Já Smunt e Watts (2003) propuseram técnicas de agregação dos dados coletados do processo, levando à redução da variância dos valores preditos pela curva.
A utilização do número de unidades produzidas, como única variável independente para o monitoramento do progresso de trabalhadores em uma tarefa, também tem gerado questionamentos quanto à eficiência dos modelos de aprendizado. Fine (1986) argumenta que unidades acumulativas podem esconder falhas graves no processo de aprendizado, pois não avaliam a qualidade da unidade produzida. $\mathrm{O}$ autor sugeriu modificações na curva, como: somente considerar as unidades-conformes na análise do processo de aprendizado. Li e Rajagopalan (1997) ampliaram a curva proposta por Fine (1986), utilizando tanto o número de unidades-conformes como o de não-conformes para o monitoramento do processo de aprendizado. Por sua vez, Jaber e Guiffrida (2004) propuseram modificações no modelo de Wright capazes de monitorar processos geradores de unidades defeituosas e submetidas ao retrabalho.

Alguns modelos de curvas de aprendizado foram desenvolvidos como extensões do modelo potencial proposto por Wright, visando sua utilização em cenários específicos. O modelo de Stanford-B:

$$
y=C_{1}(x+B)^{b}
$$

foi desenvolvido com o propósito de incorporar a experiência prévia do trabalhador ao modelo potencial. A experiência prévia é quantificada pelo parâmetro $B$, sendo sua função deslocar o desempenho do trabalhador a patamares mais elevados, através da inclusão de unidades produzidas equivalentes à experiência prévia (TEPLITZ, 1991; BADIRU, 1992; NEMBHAARD; UZUMERI, 2000a). Esse modelo foi utilizado em etapas da fabricação do Boeing 707, sendo posteriormente também aplicado na incorporação de alterações na estrutura da aeronave (YELLE, 1979; BADIRU, 1992; NEMBHARD; UZUMERI, 2000a).

O modelo de DeJong analisa a influência da participação de maquinário no processo de aprendizado. A Equação 5 é representada por:

$$
y=C_{1}\left[M+(1-M) x^{b}\right]
$$

onde, $\mathrm{M}(0 \leq M \leq 1)$ é o fator de incompressibilidade e representa a proporção do tempo total de operação constituído por procedimentos automatizados (YELLE, 1979; BADIRU, 1992). Quando $M$ assume valor zero, não existe participação de maquinário no processo e o modelo se reduz à tradicional equação de Wright. No caso de $M=1$, o processo é integralmente controlado por máquinas, não sendo possível a compressão, isto é, a diminuição através do aprendizado do tempo demandado para realização das tarefas (processos totalmente automatizados são exemplos dessa condição). (BADIRU, 1992).

A curva $S$ contempla operações que necessitam da intervenção de máquinas e cuja análise do desempenho nas primeiras unidades assume importância para 
a descrição do processo. A Equação 6 foi desenvolvida através da união dos modelos de DeJong e Stanford-B e é representada por:

$$
y=C_{1}\left[M+(1-M)(x+B)^{b}\right]
$$

onde, os parâmetros assumem o mesmo significado dos modelos em que foi baseada (BADIRU, 1992; NEMBHARD; UZUMERI, 2000a).

O modelo de Plateau é composto pelo acréscimo de uma constante aditiva ao modelo de Wright:

$$
y=C+C_{1} x^{b}
$$

fazendo com que y tenda a $\mathrm{C}$ quando o volume de produção for elevado. $\mathrm{O}$ valor da constante $C$ reproduz o desempenho do trabalhador ao atingir o estado estacionário, situação em que i) o processo de aprendizado está próximo à conclusão ou ii) existe alguma limitação imposta pelo maquinário ao progresso do trabalhador na execução da tarefa (YELLE, 1979; TEPLITZ, 1991; LI; RAJAGOPALAN, 1998).

A Figura 1 permite a comparação entre os perfis gerados pelo modelo de Wright e pelas extensões a este modelo apresentadas até aqui; a unidade de desempenho é medida na forma de tempo demandado por unidade fabricada.

Além dos modelos potenciais comumente utilizados, equações alternativas foram desenvolvidas para utilização em cenários específicos. Dada sua complexidade e o número restrito de setores possíveis de aplicação, os mesmos foram pouco explorados na literatura. Dentre esses, merece destaque a Função Adaptada de Levy:

$$
M y=\left[\frac{1}{\beta}-\left(\frac{1}{\beta}-\frac{x^{b}}{C_{1}}\right) k^{-k x}\right]^{-1}
$$

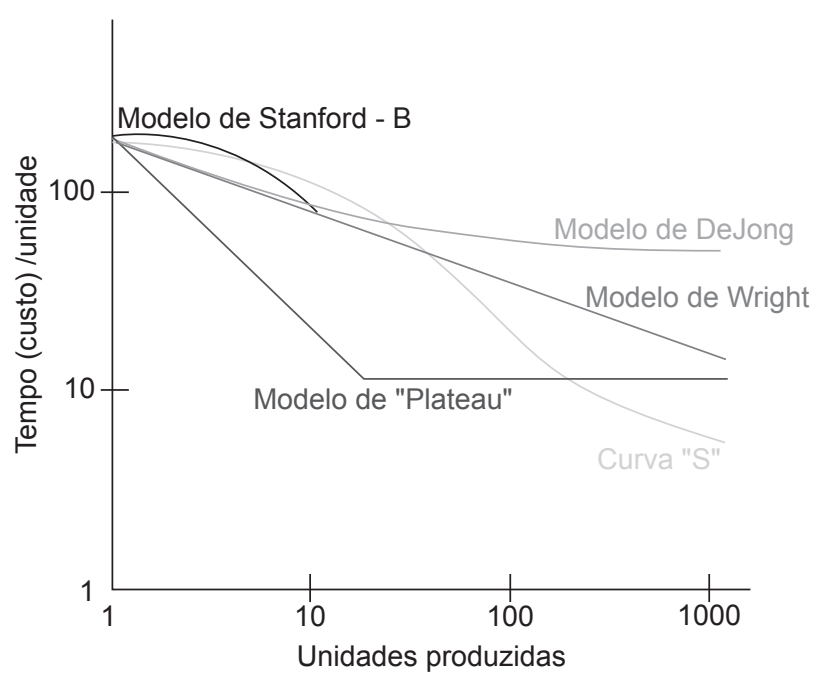

Figura 1. Comparação dos modelos de curva de aprendizado de natureza potencial em escala logarítmica. onde, $\beta$ representa o índice de produção para a primeira unidade (valor tabelado de acordo com a natureza da operação) e $k$ corresponde ao desempenho em estado estacionário (LEVY, 1965; BADIRU, 1992; NEMBHARD; UZUMERI, 2000a). Os demais parâmetros seguem as definições dos modelos anteriores.

Buscando contornar as divergências verificadas em corridas de produção consideradas longas (acima de 200 unidades), Knecht (1974) e Nembhard e Uzumeri (2000a) apresentaram um modelo capaz de retratar processos em que ocorrem modificações no valor de $b$ no decorrer da corrida de produção. O Modelo de Knecht é expresso por:

$$
y=\frac{C_{1} x^{b+1}}{(1+b)}
$$

onde os parâmetros têm significados idênticos aos modelos anteriores. Complementarmente, Yelle (1976) propôs um modelo baseado no somatório de curvas de aprendizado para os $n$ diferentes valores de $b$ assumidos pela operação (bem como para $n$ diferentes operações, caso esse fosse o objetivo da análise). O modelo assumiu a forma:

$$
y=C_{1} x_{1}^{b_{1}}+C_{2} x_{2}^{b_{2}}+\ldots+C_{n} x_{n}^{b_{n}}
$$

onde, os parâmetros mantêm os significados dos modelos anteriores. As deficiências deste modelo, no entanto, foram apontadas por Howell (1980), principalmente no que diz respeito à sua limitada precisão em aplicações práticas.

Alguns cenários específicos de produção forneceram subsídios para o desenvolvimento de curvas de aprendizado baseadas nos preceitos dos modelos potenciais clássicos, sem utilizarem tais modelos em suas metodologias. Por conta de suas complexidades matemáticas, apenas as aplicações dos modelos serão descritas. Exemplo dessa situação é o modelo sugerido por Klenow (1998), o qual se baseia em uma sistemática para a tomada de decisão em torno do aprimoramento (update) da tecnologia de produção. Demeester e Qi (2005) propuseram um modelo de curva de aprendizado para cenários caracterizados pela produção simultânea de duas gerações de um mesmo produto (antigo e recente). A curva permite definir o momento mais adequado para que todos os recursos de aprendizado (treinamentos e incentivos aos trabalhadores, dentre outros) sejam direcionados integralmente para o novo modelo de produto.

Mazzola, Neebe e Rump (1998) desenvolveram um algoritmo que utiliza curvas de aprendizado para o planejamento da produção multiproduto em ambientes caracterizados por elevados níveis de aprendizado e esquecimento. Já Gavious e Rabinowitz (2003) propuseram uma sistemática que permite comparar os resultados do treinamento da mão-de-obra interna frente 
ao desempenho de equipes terceirizadas. Com objetivos semelhantes, Fioretti (2005) utiliza um modelo desagregado de curva de aprendizado organizacional destinado a analisar características dos recursos produtivos (trabalhadores e maquinário), buscando analisar o ritmo de redução no tempo demandado para produção.

Um modelo de monitoramento de aprendizado que objetiva minimizar o tempo de atravessamento de produtos, que dependem de processamento em uma mesma máquina foi desenvolvido por Mosheiov e Sidney (2003), enquanto que Park, Lee e Kim (2003) propuseram uma curva de aprendizado multiresposta objetivando avaliar a transferência de conhecimento entre as diversas gerações do processo produtivo em uma empresa de painéis LCD (Liquid Crystal Display).

\subsection{Modelos exponenciais}

Curvas de aprendizagem de natureza exponencial são constituídas por parâmetros diferenciados, quando comparados aos modelos potenciais. Tais parâmetros permitem extrair maior quantidade de informação a respeito do processo de aprendizado do trabalhador, gerando previsões de produção mais precisas que as fornecidas pelas curvas potenciais (NEMBHARD; UZUMERI, 2000a).

Os primeiros estudos em torno dos modelos exponenciais reportam a Knecht (1974), o qual propôs a utilização combinada de funções exponenciais e potenciais, buscando aprimorar a modelagem de processos caracterizados por longa duração (elevado número de repetições). Sua proposição segue a forma:

$$
y=C_{1} x^{b} e^{c x}
$$

onde, $c$ é uma segunda constante e os demais parâmetros são idênticos aos modelos anteriores.

Dentre os modelos exponenciais mais difundidos, merecem destaque: o exponencial de três parâmetros, o exponencial de dois parâmetros e o de tempo constante. O modelo exponencial de três parâmetros é dado por:

$$
y=k\left(1-e^{-(x+p) / r}\right)
$$

em que y é o desempenho do trabalhador na execução de determinada tarefa, expresso em unidades produzidas após $x$ unidades de tempo de operação $(y \geq 0), x$ denota o tempo de operação na tarefa, expresso em unidades de tempo $(x \geq 0), k$ é o patamar máximo de desempenho a ser atingido quando a aquisição de conhecimento for integral, expresso em número de unidades produzidas por tempo de operação $(k \geq 0)$, p designa a experiência prévia do trabalhador na execução da tarefa em questão, expresso em unidades de tempo ( $p \geq 0)$, e r quantifica a taxa de aprendizado do trabalhador, expressa em unidades de tempo $(r \geq 0)$.

Estudos realizados por Mazur e Hastie (1978) mostram que o modelo apresentado na Equação 12 resulta em modelagens deficientes quando o trabalhador analisado é submetido a tarefas complexas e que demandam grande quantidade de novos conhecimentos. Em contrapartida, o modelo gera bons resultados em situações em que o trabalhador apresenta experiência prévia.

O modelo exponencial de dois parâmetros difere do anterior por não apresentar o parâmetro $p$ (experiência prévia), além de resultar em aderências menos eficientes se comparado ao modelo de três parâmetros (MAZUR; HASTIE, 1978).

O modelo de tempo constante foi desenvolvido por Towill (1990) e se baseia em uma estrutura semelhante à curva exponencial de três parâmetros, sendo dado por:

$$
y=y_{c}+y_{f}\left(1-e^{-t / \tau}\right)
$$

onde, $y_{c}$ quantifica o desempenho inicial do trabalhador (unidades/tempo) e $y_{f}$ indica o aprimoramento máximo de desempenho quando o trabalhador atingir o estado estacionário de aprendizado, também expresso em unidades por tempo. O modelo utiliza o tempo acumulado de operação $(t)$ como variável independente, sendo seu significado idêntico ao número de unidades produzidas $(x)$ adotado pelos demais modelos de curvas. Essa adaptação possibilita a determinação simplificada do tempo demandado para a obtenção de determinado patamar de desempenho. Naim e Towill (1990) adicionaram funções trigonométricas ao modelo na Equação 13, testando o modelo resultante em situações nas quais há alternância entre aprimoramento contínuo e variações cíclicas de desempenho, decorrentes de fatores conhecidos. Howell (1990) analisou as conseqüências de parâmetros mal estimados sobre a capacidade preditiva do modelo, bem como sugeriu alternativas para contornar situações em que não fosse possível alcançar convergência na modelagem.

Para Towill (1990), o modelo de tempo constante é apropriado para a modelagem de processos em que a coleta de dados de desempenho se inicia depois de um pequeno período de adaptação do trabalhador à tarefa. $\mathrm{O}$ mesmo modelo foi utilizado por Dardan, Busch e Sward (2004) para avaliar a influência do aprendizado sobre o tempo de vida de investimentos tecnológicos em uma empresa de hardware.

\subsection{Modelos hiperbólicos}

Mazur e Hastie (1978) propuseram uma formulação de curva de aprendizado baseada na razão das unidades consideradas conformes pelo número total de unidades produzidas. A forma matemática sugerida consistiu em uma curva hiperbólica de dois parâmetros (apresentada na Equação 14). Uma análise preliminar da equação, segundo seus princípios de concepção, permite associar a variável $\mathrm{x}$ ao número de unidades conformes, $r$ ao número 
de unidades não-conformes e $y$ à proporção de unidades consideradas corretas.

$$
y=k\left(\frac{x}{x+r}\right)
$$

Para fins de modelagem do desempenho de trabalhadores, os parâmetros na Equação 14 apresentam significado semelhante ao modelo exponencial de dois parâmetros, cuja variável de resposta $y$ representa o número de unidades produzidas em um intervalo de operação $x, k$ é o patamar máximo de desempenho a ser atingido e $r$ indica a taxa de aprendizado (NEMBHARD; UZUMERI, 2000a).

Mazur e Hastie (1978) reportam a adição do parâmetro $p$ ao modelo na Equação 14, buscando verificar o efeito da experiência prévia do trabalhador na execução da tarefa, gerando com isso, o modelo hiperbólico de três parâmetros, apresentado na Equação 15.

$$
y=k\left(\frac{x+p}{x+p+r}\right)
$$

Os parâmetros deste modelo assumem significado idêntico aos do modelo exponencial de três parâmetros. Uzumeri e Nembhard (1998) e Nembhard e Uzumeri (2000a) sugeriram aprimoramentos na definição do parâmetro $r$, este passou a ser tratado como o tempo de operação necessário para que o trabalhador atinja $k / 2$ (que corresponde à metade do patamar de desempenho máximo $k$ ), conforme apresentado na Figura 2. Valores elevados de $r$ indicam a necessidade de elevado tempo de operação para atingir determinado valor de $k$ (sinalizando um processo de aprendizado lento), ao passo que valores reduzidos sinalizam rápida assimilação dos preceitos da tarefa.

De acordo com Uzumeri e Nembhard (1998) e Nembhard e Uzumeri (2000a), $r$ atua como parâmetro de forma da equação hiperbólica, podendo descrever

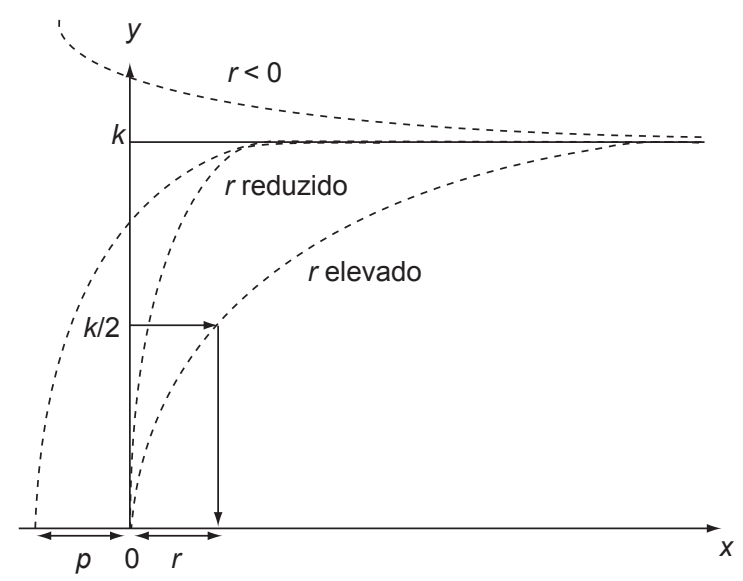

Figura 2. Perfis gerados pelo modelo hiperbólico de 3 parâmetros. três padrões distintos de aprendizado: i) $\mathrm{r}>0-\mathrm{a}$ curva cresce até um valor limite de desempenho $k$, retratando situações típicas de trabalhadores com reduzida experiência prévia submetidos a novas operações; ii) $r \rightarrow 0-a$ curva comporta-se como uma linha horizontal, descrevendo um processo no qual não há aprendizado; e iii) $\mathrm{r}<0$ - situações onde o desempenho, representado por uma curva decrescente, diminui com o transcorrer do processo (ocasionado por fadiga ou por esquecimento da tarefa). A Figura 2 apresenta os perfis gerados pelo modelo hiperbólico de três parâmetros, de acordo com o sinal e magnitude do parâmetro $r$.

A similaridade na interpretação dos parâmetros $k, r$ e $p$ das Equações 12 e 15, apesar das estruturas matemáticas distintas, levou Mazur e Hastie (1978) a desenvolverem uma série de experimentos, buscando comparar os resultados previstos pelos modelos exponencial e hiperbólico. Os parâmetros $r$ e $p$ gerados pelos modelos apresentaram grandezas similares. As maiores diferenças foram verificadas no valor de desempenho final $(k)$ estipulado pelas equações. De maneira geral, o modelo exponencial subestima o parâmetro $k$, levando a previsões de desempenho inferiores aos valores verificados em aplicações práticas, além de apresentar menor coeficiente de determinação $R^{2}$ quando comparado ao modelo hiperbólico.

Buscando avaliar o potencial do modelo descrito em Uzumeri e Nembhard (1998), Nembhard e Uzumeri (2000a) realizaram comparações entre 11 modelos de curvas de aprendizado, incluindo o modelo hiperbólico de três parâmetros. Os modelos foram testados em termos de eficiência, estabilidade, número de parâmetros e condições de modelar episódios de aprendizado negativo (esquecimento), sendo que o modelo hiperbólico de três parâmetros apresentou os melhores resultados. Anzanello (2004) e Anzanello e Fogliatto (2005, 2006) compararam o modelo hiperbólico de três parâmetros a outros dois modelos (exponencial de três parâmetros e modelo de Tempo Constante), sendo que novamente a curva hiperbólica conduziu a modelagens superiores.

Uzumeri e Nembhard (1998) e Shafer, Nembhard e Uzumeri (2001) utilizaram o modelo hiperbólico para o monitoramento dos diversos perfis de aprendizado apresentados por uma população de trabalhadores submetidos a novas operações, através da análise dos parâmetros gerados pelas modelagens. Os autores concluíram que, trabalhadores que apresentam rápida adequação à nova operação (baixo valor de $r$ ) tendem a atingir patamares de desempenho final $k$ inferiores aos dos trabalhadores que assimilam as operações de forma mais lenta (alto valor de $r$ ). De tal forma, é possível alocar trabalhadores com reduzidos valores de $r$ a operações de curta duração, enquanto que trabalhadores que apresentam elevados valores de $r$ devem ser direcionados a tarefas de maior duração, dados seus patamares elevados de 
desempenho final. Em estudo semelhante, Nembhard e Uzumeri (2000a) estenderam as conclusões obtidas em Uzumeri e Nembhard (1998), afirmando que o método proposto pode ser utilizado para a avaliação da eficácia de programas de treinamento e otimização na alocação de trabalhadores a tarefas críticas.

Nembhard e Osothsilp (2002) utilizaram o modelo hiperbólico de três parâmetros para a análise da complexidade das tarefas sobre o aprendizado de trabalhadores, servindo como subsídio para a correta alocação de trabalhadores a determinadas tarefas. Valendo-se do mesmo modelo, Nembhard e Uzumeri (2000b) analisaram perfis distintos de aquisição e retenção de conhecimento de trabalhadores submetidos a tarefas diversas, enquanto que Anzanello e Fogliatto (2005) utilizaram a curva para a otimização no direcionamento de modelos de produtos a equipes de trabalhadores em uma empresa calçadista.

\subsection{Comparativo entre modelos univariados}

A Figura 3 apresenta um resumo das características dos principais modelos de curvas de aprendizado univariadas descritas nas seções anteriores. Nessa figura, são apontados os modelos de curvas mais aptos a serem utilizados em situações específicas de modelagem, assim como em cenários onde se verifica esquecimento, e nos quais é demandada a participação de máquinas. A Figura 3 também traz uma classificação dos modelos de curvas de acordo com a unidade de medida das variáveis $y$ e $x$ (variável de resposta e variável independente, respectivamente).

\subsection{Modelos multivariados}

Modificações e extensões das tradicionais curvas de aprendizado são necessárias para uma melhor caracterização do processo de aprendizado quando se deseja analisar a influência de fatores qualitativos e quantitativos (BADIRU, 1992). Os modelos multivariados fazem uso de duas ou mais variáveis independentes, assumindo a forma genérica:

$$
C_{x}=K \prod_{i=1}^{n} c_{i} x_{i}^{b_{i}}
$$

onde, $K$ representa o desempenho (custo) da primeira unidade, $c_{i}$ é o coeficiente da variável independente $i$, e os demais parâmetros conservam as definições dos modelos potenciais anteriores. É importante mencionar que modelos multivariados, no contexto de curvas de aprendizado, referem-se a modelos com mais de uma variável independente, não estando vinculados a técnicas multivariadas de análise estatística.
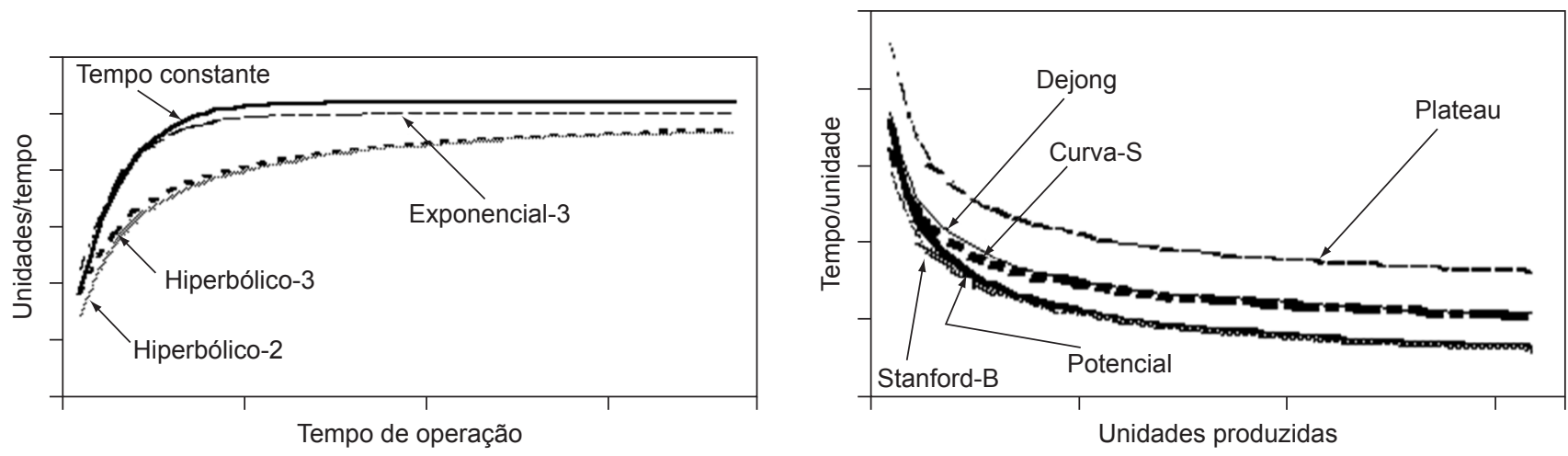

\begin{tabular}{llcccc}
\hline \multicolumn{1}{c}{ Modelo } & \multicolumn{1}{c}{ Forma } & $\mathbf{N}^{\mathbf{0}}$ parâmetros & $\begin{array}{c}\text { Modelagem de } \\
\text { esquecimento }\end{array}$ & $\begin{array}{c}\text { Experiência } \\
\text { prévia }\end{array}$ & $\begin{array}{c}\text { Participação de máquinas } \\
\text { na operação }\end{array}$ \\
\hline Hiperbólico-2 & $y=k[x /(x+r)]$ & 2 & $\sqrt{ }$ & \\
Hiperbólico-3 & $y=k[(x+p) /(x+p+r)]$ & 3 & $\sqrt{ }$ & \\
Exponencial-3 & $y=k\left(1-\mathrm{e}^{-(x+p) / r}\right)$ & 3 & & $\sqrt{ }$ \\
Tempo constante & $y=y_{c}+y_{f}\left(1-\mathrm{e}^{-x / t}\right)$ & 3 & & & \\
Potencial & $y$ & $=C x^{-b}$ & 2 & & $\sqrt{ }$ \\
Plateau & $y=B+C x^{-b}$ & 3 & & & \\
Stanford-B & $y$ & $=C(x+B)^{-b}$ & 3 & & \\
Dejong & $y$ & $=C\left[M+(1-M) x^{-b}\right]$ & 3 & $\sqrt{ }$ & $\sqrt{ }$ \\
Curva-S & $y$ & $=C\left[M+(1-M)(x+B)^{-b}\right]$ & 4 & $\sqrt{ }$ & $\sqrt{ }$ \\
\hline
\end{tabular}

Figura 3. Resumo das principais características dos modelos de curvas de aprendizado univariados. 
Quando modelos multivariados utilizam duas variáveis independentes para descrição do processo de aprendizado, é possível analisar a influência das variáveis $x_{1} \mathrm{e}$ $x_{2}$ através de gráficos de superfície, como exemplificado na Figura 4.

Estudos desenvolvidos por Gold (1981) e Camm et al. (1987) utilizaram a Equação 16 e estruturas semelhantes no monitoramento de custos de produção, avaliando a influência de variáveis independentes distintas sobre tais custos. Entre as variáveis analisadas, destacam-se: o número de unidades produzidas, a taxa de produção, o custo e a duração de programas de treinamento e a complexidade das tarefas demandadas.

Dentre os modelos de curvas de aprendizado multivariado que diferem da Equação 16, merecem destaque os sugeridos por McIntyre (1977) e Womer (1979), os quais se baseiam em procedimentos de integração. Em decorrência de suas complexidades, tais modelos apresentam aplicações práticas bastante escassas.

Badiru (1992) realizou estudos comparando o poder de predição dos modelos univariados e multivariados, concluindo que modelos multivariados fornecem informações importantes sobre a interação das variáveis independentes, mas devem ser utilizados somente quando se dispõem de informações relevantes sobre as mesmas. Caso contrário, os modelos univariados apresentamse como melhor alternativa, dada a sua simplicidade. Alerta ainda, que a adição de variáveis independentes de menor relevância a modelos multivariados pode gerar problemas quanto à estimação de seus parâmetros através de métodos de regressão. A eventual multicolinearidade

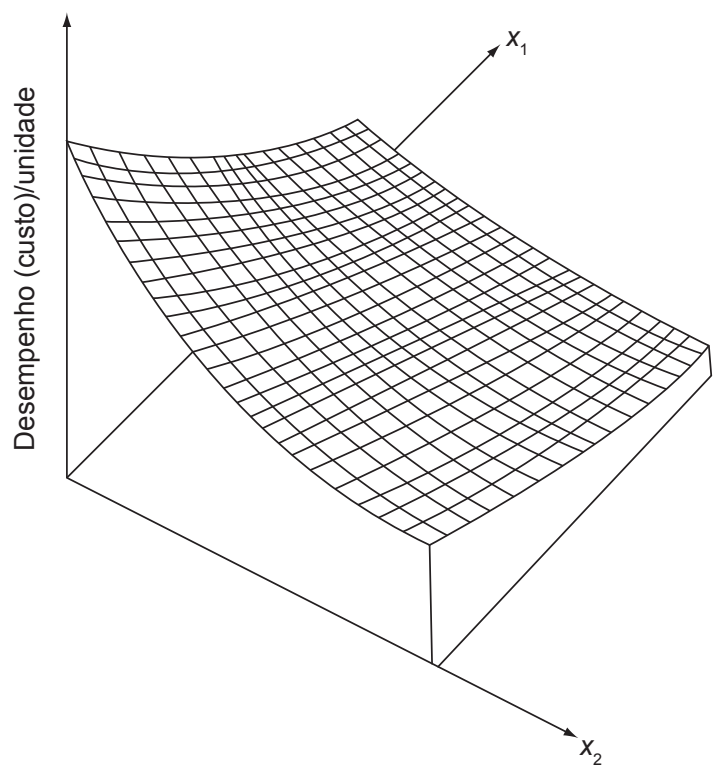

Figura 4. Gráfico genérico de uma curva de aprendizado bivariada. (interdependência) entre variáveis supostamente independentes é outra limitação dos modelos multivariados.

A opção por determinado modelo multivariado frente aos demais depende da disponibilidade de informações sobre as variáveis independentes. Na presença dessas variáveis, qualquer modelo pode ser testado, sendo sua utilização balizada pela aderência a critérios de ajuste pré-estabelecidos. Vale acrescentar, que a literatura sobre modelos multivariados de curvas de aprendizado é bastante restrita, além de estar fundamentada em estudos não muito recentes.

\subsection{Modelos de esquecimento}

A ocorrência de paradas freqüentes de produção e modificações nas especificações dos produtos originou uma série de estudos, nos quais se busca quantificar a influência desses fatores, principalmente no que se refere ao esquecimento dos trabalhadores. O esquecimento se traduz na forma i) diminuição no desempenho do trabalhador quando submetido a uma tarefa após um período de inatividade; e ii) na fabricação de produtos de qualidade inferior àqueles obtidos durante a produção contínua, principalmente nos primeiros ciclos. Globerson, Levin e Shtub (1989), Argote (1999), Dar-El e Rubinovitz (1991), Dar-El (2000) e Bailey e McIntyre (1997, 2003) afirmam que a capacidade de prever o nível de desempenho dos trabalhadores, quando há retomada das tarefas, permite uma melhor aplicação de recursos e planejamentos de produção mais precisos.

A verificação de que uma curva potencial semelhante à proposta por Wright é capaz de descrever o processo de esquecimento levou Globerson, Levin e Shtub (1989) a concluírem que os processos de aprendizado e esquecimento ocorrem de maneira bastante similar. Jaber e Bonney (1996) e Bailey e McIntyre (2003) também desenvolveram modelos capazes de descrever o fenômeno de esquecimento por uma curva potencial, enquanto que Jaber e Kher (2004) estenderam as pesquisas de Jaber e Bonney (1996), analisando a variabilidade no tempo necessário para o total esquecimento da forma de execução da tarefa. Tais modelos permitem determinar o tempo necessário para fabricação da primeira unidade quando a produção é retomada, e a estimar o tempo a partir do qual se verifica esquecimento total por parte do trabalhador em relação a uma tarefa. Os resultados obtidos por Jaber e Bonney (1996) e Globerson, Levin e Shtub (1989) foram comparados, verificando-se diferenças desprezíveis entre as predições dos dois modelos. Objetivando aprimorar a precisão da análise, Jaber e Bonney (1997) sugeriram três modelos matemáticos voltados para a descrição dos processos de aprendizado e esquecimento, enquanto que Baile e McIntyre (1997) idealizaram o conceito de curva de reaprendizado, baseada em modificações no modelo 
potencial e ideal para o monitoramento de processos com paradas e retomadas freqüentes de produção.

A influência do esquecimento sobre preceitos de programação da produção foi inicialmente estudada por Salameh, Abdul-Malak e Jaber (1993) e Jaber e Bonney (1996), os quais analisaram o efeito de paradas sobre o tamanho do lote ótimo de produção e o custo de estoque. Segundo Jaber e Bonney (1996, 2003), o lote ótimo de produção sofre redução contínua em seu tamanho quando se verifica transmissão integral de conhecimento, ou seja, situações onde exista produção seqüencial de lotes sem paradas longas, com set up reduzido.

A modificação de parâmetros de determinado processo ou produto durante sua vida útil, de forma análoga às paradas de produção, acarreta consideráveis diminuições no desempenho dos trabalhadores (YELLE, 1979). Tais modificações geralmente provêm de exigências de consumidores ou de adequação do produto às novas normas ou mercados, o que representam custos com reprojeto, retrabalho e aprendizado por parte dos trabalhadores envolvidos (visto que cada pequena alteração de procedimento demanda mais tempo para conclusão da tarefa). Eden, Willians e Ackermann (1998) e Lam, Lee e Hu (2001) também analisaram o efeito do esquecimento e de modificações de parâmetros em operações de construção civil, avaliando seu impacto nos indicadores produtivos.

Estudos acerca do mecanismo de esquecimento e suas conseqüências para o processo produtivo também podem ser encontrados em Nembhard e Uzumeri (2000a), Shafer, Nembhard e Uzumeri (2001) e Nembhard e Osothsilp (2002), os quais utilizaram o modelo hiperbólico de três parâmetros em suas análises.

\section{Perspectivas de pesquisa sobre curvas de aprendizado}

Curvas de aprendizado ressurgem como importante tema de pesquisa em Engenharia de Produção, motivado principalmente por dois eventos. O primeiro é o surgimento da Customização em Massa $(\mathrm{CM})$ como importante estratégia de produção. Pela lógica da $\mathrm{CM}$, produtos e serviços são personalizados de forma a atenderem necessidades individuais de clientes. Tal personalização esbarra na inflexibilidade, que caracteriza sistemas produtivos intensivos na utilização de mão-de-obra. Nesses ambientes, a modelagem de curvas de aprendizado surge como uma ferramenta de otimização da alocação de tarefas a indivíduos ou equipes de trabalho, possibilitando a produção em pequenos lotes. $\mathrm{O}$ segundo evento motivador do incremento das pesquisas sobre curvas de aprendizado é a maior difusão de métodos estatísticos multivariados e sua adaptação a aplicações específicas de Engenharia. Tais métodos permitem uma melhor mode- lagem e compreensão do mecanismo de aprendizado na realização de tarefas.

Os direcionamentos futuros de pesquisa sobre curvas de aprendizado listados na seqüência exploram aspectos relacionados a esses dois eventos. As quatro primeiras subseções apresentam propostas de pesquisa focadas no uso de curvas de aprendizado como ferramenta habilitadora da CM. As duas subseções finais trazem propostas de aplicação de técnicas multivariadas na modelagem de curvas de aprendizado.

\subsection{Metodologia para elaboração de menus de opções (choice menus) para configuração de produtos e serviços em ambientes de alta customização}

Contextos de elevada customização pressupõem produção de grande variedade de itens e alteração constante no tamanho dos lotes de produtos demandados, exigindo flexibilidade dos recursos e interação dos meios produtivos (maquinário e mão-de-obra) para se adaptarem de maneira rápida e eficiente às modificações impostas (DA SILVEIRA et al., 2001). De tal forma, estimativas dos efeitos da adaptação dos trabalhadores a novos modelos de produtos resultantes da customização, obtidas anteriormente no início do processo produtivo, constituem importante subsídio para a administração da produção.

Uma relevante contribuição em torno das curvas de aprendizado viria do desenvolvimento de uma metodologia voltada para a tomada de decisão quanto à aceitação de um novo pedido de produção e a conseqüente ampliação do menu de opções de configuração do produto ou serviço em questão. As curvas de aprendizado permitiriam prever os impactos da entrada de tais itens no sistema produtivo e, complementarmente, poderiam gerar estimativas de prazos de entrega dos lotes de produção.

\subsection{Apoio ao seqüenciamento da produção}

Sistemas produtivos intensivos em mão-de-obra, como é o caso de linhas de montagem de produtos, não costumam apresentar problemas significativos de seqüenciamento. Isto se justifica pela lógica da concepção de layouts lineares, voltados à produção de itens com demandas similares em termos de processamento. Entretanto, na medida em que a produção se diversifica para atender a demanda por produtos customizados, o problema do seqüenciamento passa a exercer um papel significativo sobre a eficiência de sistemas lineares de produção.

Curvas de aprendizado podem ser utilizadas para modelar o impacto da entrada de um novo item na seqüência de produção sobre a eficiência de indivíduos, condicionado ao item anteriormente em produção. Os modelos de aprendizado teriam que ser adaptados para que se considerassem não apenas a experiência prévia 
do indivíduo na realização da tarefa, mas o impacto da produção prévia de um determinado modelo de produto sobre a produtividade do trabalhador. Em outras palavras, a análise das características do produto que vinha sendo fabricado e sua influência sobre a adaptação do trabalhador ao modelo seguinte também se configura como interessante desdobramento da pesquisa sobre curvas de aprendizado aplicadas a ambientes de alta customização.

\subsection{Otimização na rotação de trabalhadores em linhas de montagem}

Em determinados sistemas produtivos, particularmente em linhas de montagem, os trabalhadores apresentam-se "engessados" na execução de uma operação específica (ou em um posto de trabalho fixo), nos quais são considerados peritos, independente das alterações nos modelos de produtos demandados. Em um estudo futuro, as curvas de aprendizado podem ser utilizadas para otimizar a rotação de integrantes de uma mesma linha de acordo com os modelos de produtos solicitados. Tal rotação promoveria i) benefícios em termos ergonômicos (redução da ocorrência de lesões por esforço repetitivo); ii) elevação do nível de conhecimento e destreza dos trabalhadores, servindo como treinamentos complementares; e iii) forneceria à gerência maior conhecimento do perfil dos trabalhadores envolvidos no processo.

Em uma segunda etapa, trabalhadores de várias equipes (ou linhas) poderiam ser rotados entre as equipes, obedecendo as características dos modelos de produtos demandados, buscando-se minimizar as perdas por troca de modelo e aumentar a flexibilidade dos recursos humanos.

\subsection{Otimização no desenvolvimento de produtos submetidos a procedimentos de montagem}

Os esforços no desenvolvimento de produtos, tradicionalmente, têm sido focados em ajustes robustos de equipamentos e especificações, de maneira a atender prérequisitos de custo e qualidade. No entanto, percebe-se uma escassez de pesquisas voltadas à análise dos efeitos dos procedimentos de montagem demandados por tais produtos, particularmente quando intervenções manuais são exigidas. Tendo-se em vista que, os procedimentos de montagem e as demais características do produto devem ser otimizados de maneira conjunta, sugere-se a inclusão de curvas de aprendizado nas tradicionais metodologias de desenvolvimento de produto.

A metodologia resultante permitiria projetar produtos com características otimizadas frente aos procedimentos de montagem posteriormente demandados na linha de produção, levando à redução das perdas por refugo e à elevação dos índices de produtividade e de qualidade dos produtos obtidos nos primeiros ciclos de produção.

\subsection{Aplicação da análise de conglomerados em curvas de aprendizado para formação de células homogêneas de trabalhadores}

Em linhas de montagem, é comum a alocação de vários indivíduos em um mesmo posto de trabalho, visando atingir uma determinada capacidade produtiva. Usualmente, indivíduos são agrupados segundo seus tempos médios de realização das tarefas alocadas ao posto; o objetivo é agrupar trabalhadores minimizando a variância de seus tempos médios, permitindo uma distribuição homogênea da carga de trabalho entre os trabalhadores no posto. Tais tempos médios são normalmente calculados após ser concluído o período de aprendizado na realização das tarefas.

A análise funcional de conglomerados tem por objetivo a identificação de grupos de indivíduos similares, a partir da análise de variáveis com realizações dadas na forma de perfis no lugar dos valores pontuais. Esse tipo de análise, objeto de estudo de Heckman e Zamar (2000) e Tarpey e Kinateder (2003), dentre outros, ainda é pouco explorada nos estudos de Engenharia.

A análise funcional de conglomerados usando curvas de aprendizado teria por objetivo otimizar a alocação de indivíduos em postos de trabalho, considerando seu desempenho na execução de tarefas desde o seu início, e não somente após o atingimento de um nível estacionário de produção. Em cenários de produção em lotes de tamanho reduzido, a formação de conglomerados de trabalhadores (considerando seus perfis de aprendizado) é potencialmente mais interessante que o agrupamento baseado em tempos médios de execução de tarefas.

\subsection{Aplicação da análise de regressão multivariada para obtenção de curvas de aprendizado que caracterizem equipes de trabalhadores}

$\mathrm{Na}$ modelagem de curvas de aprendizado, ajustamse modelos não-lineares a dados empíricos, através de heurísticas de estimação de parâmetros. Trata-se de uma modelagem univariada, baseada na análise de uma única variável dependente, associada ao desempenho do trabalhador (ou do desempenho médio de grupos de trabalhadores) desempenhando uma tarefa.

A otimização da alocação de produtos a linhas de produção deveria, em teoria, considerar o desempenho médio da linha, levando em conta todos os seus postos de trabalho. Para tanto, a alocação deve basear-se em modelos multivariados de aprendizado, nos quais perfis de aprendizado obtidos de diferentes postos de trabalho na linha são considerados conjuntamente na obtenção de um modelo que caracterize o desempenho da linha.

Adicionalmente, poderia-se explorar o uso de pesos de importância aos perfis de aprendizado oriundos de diferentes postos de trabalho, de forma a gerar modelos 
multivariados mais influenciados pelo desempenho de trabalhadores em postos-chave.

\section{Conclusões}

O estudo da curva de aprendizado tem despertado interesse de setores industriais e acadêmicos nos últimos 80 anos. Neste período, a ferramenta foi utilizada nos mais diversos processos dos setores industriais e de serviços, o que levou ao desenvolvimento de um considerável conjunto de modelos de curvas. Entre suas principais utilizações, destacam-se: a programação da produção, a alocação de recursos produtivos, as estimativas de prazos de conclusão de tarefas e o monitoramento de custos associados à produção.

Este artigo apresenta uma revisão da literatura em torno do tema, contemplando a origem e a estruturação matemática dos modelos existentes, bem como as aplicações em contextos práticos e as limitações decorrentes das particularidades dos sistemas modelados. Tais limitações forneceram subsídios para modificações e aperfeiçoamento dos tradicionais modelos, levando a uma geração de curvas mais robustas e flexíveis. Por fim, foram apresentados direcionamentos para pesquisas futuras, embasados nas lacunas ainda existentes em torno do tema, a partir de recentes desenvolvimentos na área de Engenharia de Produção.

\title{
Learning curves: literature review and further research
}

\begin{abstract}
Learning curves have been proven to be effective tools for monitoring the performance of workers exposed to a new task, describing their development as task repetition takes place. These curves were originally proposed by Wright in 1936, and have been used since then to evaluate the time demanded to complete production runs, to estimate production cost reduction as learning takes place, and to assign workers to tasks based on their performance profile. This paper presents the state-of-the-art in the literature on learning curves, covering the existing models, their limitations, and reported applications. Further research on the subject is proposed.
\end{abstract}

Keywords: Learning curves. Workers performance. Production planning.

\section{Referências bibliográficas}

ADLER, P. S.; CLARK, K. B. Behind the Learning Curve: A Sketch of the Learning Process. Management Science, Evanston, v. 37, n. 3, p. 267-281, 1991.

AGRELL, P. J.; BOGETOFT, P.; TIND, J. Incentive Plans for Productive Efficiency, Innovation and Learning. International Journal of Production Economics, New York, v. 78, n. 1, p. 1-11, 2002.

ANDERSON, J. R. Acquisition of Cognitive Skill. Psychological Review, Washington, v. 89, n. 4, p. 369-406, 1982.

ANZANELlO, M. Curvas de Aprendizado como Balizadoras da Alocação de Modelos de Produtos a Equipes de Trabalhadores. 2004. 136 f. Dissertação (Mestrado em Engenharia de Produção) - Programa de Pós-Graduação em Engenharia de Produção, UFRGS, Porto Alegre, 2004.

ANZANELLO, M.; FOGLIATTO, F. Alocação de modelos de produtos a equipes de trabalhadores baseada em modelos de curvas de aprendizagem. Revista Produção, São Paulo, v. 15, n. 2, p. 221-234, 2005.

ANZANELLO, M.; FOGLIATTO, F. Learning curve modeling of work assignment in mass customized assembly lines. International Journal of Production Research, London, Aguardando publicação, 2006.
ARGOTE, L. Organizational Learning: Creating, Retaining and Transferring Knowledge. New York: Springer-Verlag, 1999.

ASKIN, R.; GOLDBERG, J. Design and Analysis of Lean Production Systems. New York: John Wiley \& Sons, 2001.

BADIRU, A. B. Computational Survey of Univariate and Multivariate Learning Curve Models. IEEE Transactions on Engineering Management, New Jersey, v. 39, n. 2, p. 176-188, 1992.

BAILEY, C. D.; McINTYRE, E. V. The Relation between Fit and Prediction for Alternative Forms of Learning Curves and Relearning Curves. IIE Transactions, London, v. 29, n. 6, p. 487-495, 1997.

BAILEY, C. D.; McINTYRE, E. V. Using Parameter Prediction Models to Forecast Post-Interruption Learning. IIE Transactions, London, v. 35, n. 1, p. 1077-1090, 2003.

BLANCETT, R. S. Learning from Productivity Learning Curves. Research-Technology Management, Arlington, May-June 2002.

CAMM, J. D.; EVANS, J. R.; WOMER, N. K. The Unit Learning Curve Approximation of Total Costs. Computers and Industrial Engineering, Netherlands, v. 12, n. 3, p. 205-213, 1987. 
CHAMBERS, S.; JOHNSTON, R. Experience Curves in Services: Macro and Micro Level Approaches. International Journal of Operations \& Production Management, London, v. 20, n. 7, p. $842-859,2000$.

COOK, J. A Competitive Model of the Japanese Firm, Journal of Policy Modeling, New York, v. 13, n. 1, p. 93-114, 1991.

COX, W. E. Product Life Cycles as Marketing Models. Journal of Business, Chicago, v. 40, p. 375-384, oct. 1967.

DA SILVEIRA, G.; BORESTEIN, D.; FOGLIATTO, F. S. Mass Customization: Literature Review and Research Directions. International Journal of Production Economics, New York, v. 72, n. 1, p. 1-13, 2001.

DARDAN, S.; BUSCH, D.; SWARD, D. An Application of the Learning Curve and the Nonconstant-Growth Dividend Model: IT Investment Valuation at Intel Corporation, Decision Support Systems, Netherlands, aguardando impressão, 2004.

DAR-EL, E. Human Learning: from Learning Curves to Learning Organizations, New York: Springer, 2000.

DAR-EL, E. M.; RUBINOVITZ, J. Using Learning Theory in Assembly Lines for New Products. International Journal of Production Economics, New York, v. 25, n. 1-3, p. 103-109, 1991.

DEMEESTER, L.; QI, M. Managing Learning Resources for Consecutive Product Generations, International Journal of Production Economics, New York, v. 95, n. 1, p. 265-283, 2005.

EDEN, C.; WILLIANS, T.; ACKERMANN, F. Dismantling the Learning Curve: the Role of Disruptions on the Planning of Development Projects. International Journal of Project Management, Netherlands, v. 16, n. 3, p. 131-138, 1998.

EYRING, J. D.; JOHNSON, D. S.; FRANCIS, D. J. A Cross-Level Units-of-Analysis Approach to Individual Differences in Skill Acquisition. Journal of Applied Psychology, Washington, v. 78, n. 5, p. 805-814, 1993.

FINE, C. H. Quality Improvement and Learning in Productive Systems. Management Science, Evanston, v. 10, n. 10, p. 1301-1315, 1986.

FIORETTI, G. The Organizational Learning Curve, European Journal of Operational Research, Netherlands, aguardando publicação, 2005.

FRANCESCHINI, F.; GALETTO, M. Asymptotic Defectiveness of Manufacturing Plants: an Estimate Based on Process Learning. International Journal of Production Research, London, v. 40, n. 3, p. 537-545, 2002.

GARVIN, D. Learning in Action: A guide to put the learning organization to work, Boston: Harvard Business School Press, 2000.

GAVIOUS, A.; RABINOWITZ, G. Optimal Knowledge Outsourcing Model, Omega, Netherlands, v. 31, n. 6, p. 451-457, 2003.

GLOBERSON, S. The Deviation of Actual Performance around Learning Curve Models. International Journal of Production Research, London, v. 22, n. 1, p. 51-62, 1984.

GLOBERSON, S.; GOLD, D. Statistical Attributes of the Power Learning Curve Model. International Journal of Production Research, London, v. 35, n. 3, p. 699-711, 1997.

GLOBERSON, S.; LEVIN, N. Incorporating Forgetting into Learning Curves. International Journal of Production Management, London, v. 7, n. 4, p. 80-94, 1987.

GLOBERSON, S.; LEVIN, N.; SHTUB, A. The Impact of Breaks on Forgetting When Performing a Repetitive Task. IIE Transactions, London, dec. 1989.
GRUBER, H. Learning by Doing and Spillovers: Further Evidence for the Semiconductor Industry, Review of Industrial Organization, Netherlands, v. 13, n. 6, p. 679-711, 1998.

GRUBER, H. The Yield Factor and the Learning Curve in Semiconductor Production. Applied Economics, London, v. 26, n, 8, p. 837-843, 1994.

GRUBER, H. Trade Policy and Learning by Doing: the case of Semiconductors, Research Policy, Netherlands, v. 25, p. 723-739, 1996.

HECKMAN, N. E.; ZAMAR, R. H. Comparing the shapes of regression functions, Biometrika, London, v. 87, n. 1, p. 135144, 2000.

HOWELL, S. Learning Curves for New Products, Industrial Marketing Management, Netherlands, v. 9 n. 2, p. 97-99, 1980.

HOWELL, S. Parameter Instability in Learning Curve Models: Invited Comments on Papers by Towill and by Sharp and Price, International Journal of Forecasting, Netherlands, v. 6, n. 4, p. 541-547, 1990.

HURLEY, J. W. When Are We Going to Change the Learning Curve Lecture? Computers \& Operations Research, Netherlands, v. 23, n. 5, p. 509-511, 1996.

JABER, M. Y.; BONNEY, M. A Comparative Study of Learning Curves with Forgetting. Applied Mathematical Modeling, New York, v. 21, n. 8, p. 523-531, 1997.

JABER, M. Y.; BONNEY, M. Economic Lot Sizing with Learning and Continuous Time Discounting: Is it Significant? International Journal of Production Economics, New York, v. 71, n. 1, p. 135-143, 2001.

JABER, M. Y.; BONNEY, M. Lot Sizing with Learning and Forgetting in set-ups and in Production Quality. International Journal of Production Economics, New York, v. 83, n. 1, p. 95-111, 2003.

JABER, M. Y.; BONNEY, M. Production Breaks and the Learning Curve: the Forgetting Phenomenon. Applied Mathematics Modelling, New York, v. 20, n. 20, p. 162-169, 1996.

JABER, M. Y.; BONNEY, M. The Economic Manufacture/Order Quantity (EMQ/EOQ) and the Learning Curve: Past, Present, and Future. International Journal of Production Economics, New York, v. 59, n. 1-3, p. 93-102, 1999.

JABER, M.; GUIFFRIDA, A. Learning Curves for Process Generating Defects Requiring Reworks, European Journal of Production Research, Netherlands, v. 159, n. 1, p. 663-672, 2004

JABER, M.; KHER, H. Variant versus Invariant Time to Total Forgetting: the Learn-Forget Curve Model Revisited, Computers \& Industrial Engineering, Netherlands, v. 46, p. 697-705, 2004.

JACINTO, A. A. Estudo da curva de aprendizagem em uma indústria exportadora de calçados. 2001. Monografia (Graduação em Administração com habilitação em Comércio Exterior) Lajeado: Univates - Centro Universitário, 2001.

KANFER, R. Motivation and Individual Differences in Learning: an Integration of Developmental, Differential and Cognitive Perspectives. Learning and Individual Differences, Netherlands, v. 2, n. 2, p. 221-239, 1990.

KANNAN, V.; PALOCSAY, S. Cellular vs Process Layouts: an Analytic Investigation of the Impact of Learning on Shop Performance, Omega, Netherlands, v. 27, n. 5, p. 583-592, 1999.

KARAOZ, M.; ALBENI, M. Dynamic Technological Learning Trends in Turkish Manufacturing Industries, Technological 
Forecasting and Social Change, Netherlands, v. 72, n. 7, p. 866-885, 2005.

KLENOW, P. Learning Curves and the Cyclical Behavior of Manufacturing Industries, Review of Economic Dynamics, Netherlands, v. 1, n. 2, 531-550, 1998.

KNECHT, G. Costing, Technological Growth and Generalized Learning Curves, Operations Research Quart., Berlin, v. 25, n. 3, p. 487-491, sep. 1974.

KOPCSO, D.; NEMITZ, W. Learning Curves and Lot Sizing for Independent and Dependent Demand, Journal of Operations Management, Netherlands, v. 4, n. 1, p. 73-83, 1983.

KORTGE, G. Link Sales Training and Product Life Cycles, Industrial Marketing Management, Netherlands, v. 22, n. 3, p. 239-245, 1993.

KORTGE, G. et al. Linking Experience, Product Life and Learning Curves, Calculating the Perceived Value Price Range, Industrial Marketing Management, Netherlands, v. 23, n. 3, p. 221-228, 1994.

KOULAMAS, C. Quality Improvement through Product Redesign and the Learning Curve, Omega, Netherlands, v. 20, n. 2, p. 161-168, 1992.

LAM, K; LEE, D.; HU, T. Understanding the Effect of the Learning-Forgetting Phenomenon to Duration of Projects Construction. International Journal of Project Management, London, v. 19, n. 1, p. 411-420, 2001.

LEITE, M. O. A utilização das curvas de aprendizagem no planejamento da construção civil. 2002. 87 f. Dissertação (Mestrado em Engenharia de Produção) - Programa de PósGraduação em Engenharia de Produção, UFSC, Florianópolis, 2002.

LEVY, F. Adaptation in the Production Process, Management Science, Evanston, v. 11, n. 6, p. 136-154, 1965.

LI, G.; RAJAGOPALAN, S. A Learning Curve Model with Knowledge Depreciation. European Journal of Operational Research, Netherlands, v. 105, n. 1, p. 143-154, 1998.

LI, G.; RAJAGOPALAN, S. The Impact of Quality on Learning. Journal of Operations Management, Netherlands, v. 15, n. 3, p. 181-191, 1997.

MAZUR, J. E.; HASTIE, R. Learning as Accumulation: a Reexamination of the Learning Curve. Psychological Bulletin, Washington DC, v. 85, n. 6, p. 1256-1274, 1978.

MAZZOLA, J; NEEBE, A.; RUMP, C. Multiproduct Production Planning in the Presence of Work-Force Learning, European Journal of Operational Research, Netherlands, v. 106, n. 2, p. 336-356, 1998.

McINTYRE, E. Cost-Volume-Profit Analysis Adjusted for Learning. Management Science, Evanston, v. 24, n. 2, p. 149-160, 1977.

MOSHEIOV, G.; SIDNEY, J. Scheduling with General JobDependent Learning Curves, European Journal of Operational Research, Netherlands, v. 147, n. 3, p. 665-670, 2003.

MUTH, E. J.; SPREMANN, K., Learning Effect in Economic Lot Sizing. Management Science, Evanston, v. 29, n. 2, p. 102-108, 1983.

NAIM, M. M.; TOWILL, D. R. An Engineering Approach to LSE Modeling of Experience Curves in the Electricity Supply Industry. International Journal of Forecasting, Netherlands, v. 6, n. 4 , p. 549-556, 1990.

NATTER, M. et al. The Effect of Incentive Schemes and Organizational Arrangements on the New Product Development Process. Management Science, Evanston, v. 47, n. 8, p. 1029-1045, 2001.
NEMBHARD, D. A.; OSOTHSILP, N. Task Complexity Affects on Between-Individual Learning/Forgetting Variability. International Journal of Industrial Ergonomics, London, v. 29, n. 5, p. 297-306, 2002.

NEMBHARD, D. A.; UZUMERI, M. V. An Individual-Based Description of Learning within an Organization. IEEE Transactions on Engineering Management, New Jersey, v. 47, n. 3, p. 370-378, 2000a.

Experimental Learning and Forgetting for Manual and Cognitive Tasks. International Journal of Industrial Ergonomics, London, v. 25, n. 3, p. 315-326, 2000 b.

PANANISWAML, S.; BISHOP, R. C. Behavioral Implications of the Learning Curve for Production Capacity Analysis. International Journal of Production Economics, New York, v. 24, n. 1-2, p. 157-163, 1991.

PARK, S.; LEE, J.; KIM, T. Learning by Doing and Spillovers: an Empirical Study on the TFT-LCD Industry, IEEE, New Jersey, 2003.

PRAMONGKIT, P.; SHAWYUN, T.; SIRINAOVAKUL, B. Analysis of Technological Learning for the Thai Manufacturing Industry. Technovation, Netherlands, v. 20, n. 4, p. 189-195, 2000.

PRAMONGKIT, P.; SHAWYUN, T.; SIRINAOVAKUL, B. Productivity Growth and Learning Potentials of Thai Industry. Technological Forecasting \& Social Change, Netherlands, v. 69, n. 1, p. $89-101,2002$.

PRATSINI, E. The Capacitated Dynamic Lot Size Problem with Variable Technology, Computers \& Industrial Engineering, Netherlands, v. 38, n. 4, p. 493-504, 2000.

RACHAMADUGU, R.; TAN, C. Policies for Lot Sizing with Setup Learning. International Journal of Production Economics, New York, v. 48, n, 2, p. 157-165, 1997.

REA, P.; KERZNER, H. Strategic Planning: A Practical Guide, New York: John Wiley and Sons, 1997.

RINK, D. R.; SWAN, J. E. Product Life Cycle Research: a Literature Review. Journal of Business Research, Netherlands, v. 7, n. 3, p. 219, 1979.

SALAMEH, M. K.; ABDUL-MALAK, M. U.; JABER, M. Y. Mathematical Modelling of the Effect of Human Learning in the Finite Production Inventory Model. Applied Mathematical Modeling, New York, v. 17, p. 613-615, 1993.

SARASWAT, S. P.; GORGONE, J. T. Organizational Learning Curve in Software Installation: an Empirical Investigation. Information \& Management, Netherlands, v. 19, n. 1, p. 53-59, 1990.

SEREL, D. A. et al. Investing in Quality Under Autonomous and Induced Learning. IIE Transactions, London, v. 32, p. $545 ¥ 555$, June 2003 .

SHAFER, S.; NEMBHARD, D. A.; UZUMERI, M. V. The Effects of Worker Learning, Forgetting and Heterogeneity on Assembly Line Productivity. Management Science, Evanston, v. 47, n. 12, p. 1639-1653, 2001.

SMUNT, T. L. Log-Linear and Non-Log-Linear Learning Curve Models for Production Research and Cost Estimation. International Journal of Production Research, London, v. 37, n. 17, p. 3901-3911, 1999.

SMUNT, T. L.; WATTS, C. A. Improving Operations Planning with Learning Curves: Overcoming the Pitfalls of "Messy" Shop Floor Data. Journal of Operations Management, Netherlands, v. 21, n. 1, p. 93-107, 2003.

STURM, R. Cost and Quality Trends under Managed care: is there a Learning Curve in Behavior Health Carve-out Plans? Journal 
of Health Economics, Netherlands, v. 18, n, 5, p. 593-604, 1999.

TARPEY, T.; KINATEDER, K. K. Clustering functional data. Journal of Classification, Netherlands, v. 20, p. 93-114, 2003.

TENG, J.; THOMPSON, G. Optimal Strategies for General PriceQuality Decision Models of New Products with Learning Production Costs, European Journal of Operational Research, Netherlands, v. 93, n. 3, p. 476-489, 1996.

TEPLITZ, C. J. The Learning Curve Deskbook: A reference Guide to Theory, Calculations and Applications. New York: Quorum Books, 1991.

TERWIESCH, C.; BOHN, R. Learning and Process Improvement During Production Ramp-Up. International Journal of Production Economics, New York, v. 70, n. 1, p. 1-19, 2001.

TOWILL, D. R. Forecasting Learning Curves. International Journal of Forecasting, Netherlands, v. 6, n. 1, p. 25-38, 1990.

TOWILL, D. R. Management Systems Applications of Learning Curves and Progress Functions. Engineering Costs and Production Economics, Netherlands, v. 9, p. 369-383, 1985.

UZUMERI, M.; NEMBHARD, D. A Population of Learners: A New Way to Measure Organizational Learning. Journal of Operations Management, Netherlands, v. 16, n. 5, p. 515-528, 1998.

VIGIL, D. P.; SARPER, H. Estimating the Effects of Parameter Variability on Learning Curve Model Predictions. International Journal of Production Economics, New York, v. 34, n. 2, p. $187-200,1994$.
VITS, J.; GELDERS, L. Performance Improvement Theory. International Journal of Production Economics, New York, v. 77, n. 3, p. 285-298, 2002.

WATERWORTH, C. J. Relearning the Learning Curve: a Review of the Derivation and Applications of Learning-Curve Theory. Project Management Journal, Pennsylvania, v. 31, n. 1, p. 24-31, March 2000.

WICKENS, C. D.; GORDON, S. E.; LIU,Y. An Introduction to Human Factors Engineering. New York: Addison Wesley Longman Inc., 1998.

WOMER, N. Learning Curves, Production Rate and Program Costs. Management Science, Evanston, v. 25, n. 4, p. 312-319, 1979.

WRIGHT, T. P. Factors Affecting the Cost of Airplanes. Journal of the Aeronautical Sciences, London, v. 3, p. 122-128, 1936.

YELLE, L. E. Adding Life Cycles to Learning Curves. Long Range Planning, London, v. 16, n. 6, p. 82-87, 1983.

YELLE, L. E. Learning Curves: Interaction of Marketing and Production. Industrial Marketing Management, Netherlands, v. 9, n. 2, p. 311-318, 1980.

YELLE, L. E. The Learning Curve: Historical Review and Comprehensive Survey. Decision Science, London, v. 10, n. 2 , p. 302-328, 1979.

YELLE, L. Estimating Learning Curves for Potencial Products, Industrial Marketing Management, Netherlands, v. 5, n. 2-3, p. 147-154, June of 1976.

ZANGWILL, W. I.; KANTOR, P. K. The Learning Curve: a New Perspective. International Transactions in Operational Research, London, v. 7, n. 6, p. 595-607, 2000.

\section{Sobre os autores}

\section{Michel José Anzanello}

\section{Flávio Sanson Fogliatto}

Escola de Engenharia, Departamento de Engenharia de Produção, Universidade Federal do Rio Grande do Sul - UFRGS, Av. Osvaldo Aranha, 99, $5^{\circ}$ andar, CEP 90040-020, Porto Alegre, RS, Brasil

e-mail: ffogliatto@producao.ufrgs.br 
